\title{
INTERACTIVE CONTRAST ENHANCEMENT BY HISTOGRAM WARPING
}

\author{
Mark Grundland and Neil A. Dodgson \\ Computer Laboratory, University of Cambridge \\ Cambridge, United Kingdom \\ Mark@Eyemaginary.com
}

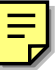

Abstract: We present an interactive contrast enhancement technique for the global histogram modification of images. Through direct manipulation, the user adjusts contrast by clicking on the image. Contrast around different key tones can be adjusted simultaneously and independently without altering their luminance. Histogram warping by monotonic splines performs the gray level mapping. User interfaces for contrast correction find application in digital photography, remote sensing, medical imaging, and scientific visualization.

Keywords: Image editing, interactive image enhancement, contrast enhancement, histogram modification, gray level transformation function, monotonic splines.

I don't paint things. I only paint the difference between things.

- Henri Matisse (1869-1954)

\section{INTRODUCTION}

Contrast is an organizing principle of visual communication, serving to bring the visual structure of information into focus. Perception draws distinctions between objects from differences between intensities. For the viewer, contrast attracts attention. For the artist, contrast conveys emphasis. In graphic design, as a way of making the first impression, contrast is used to tell the eye where to go. When presenting information, contrast makes the composition legible. When a picture carries the message, contrast is often applied to underline it. As an accent or an overstatement, contrast engages the viewer's interest. In imaging, contrast reflects a necessary compromise, 


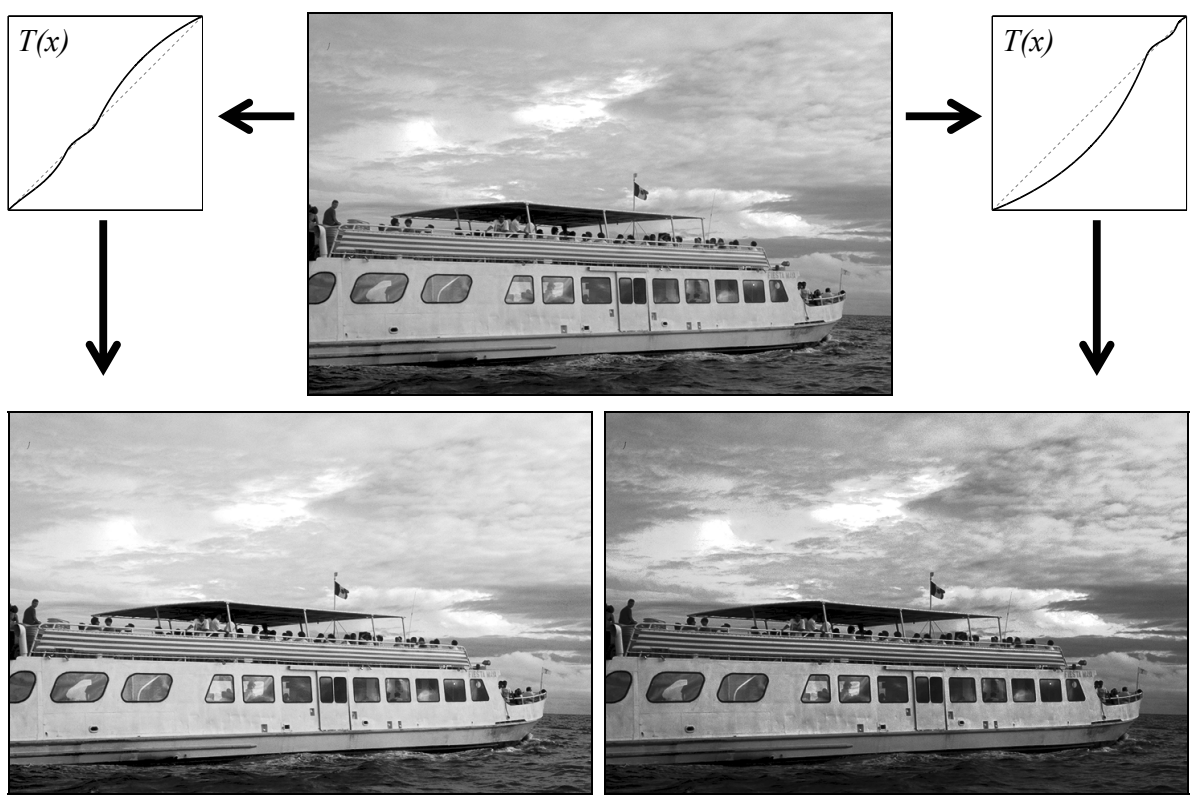

Figure 1. By adjusting contrast, the user can emphasize different aspects of the same image.

since the human visual system accommodates a dynamic range that is several orders of magnitude greater than the ones available to image reproduction systems. For a photograph to recreate the visual impression of a natural scene, the balance between light and dark tones often requires careful adjustment. For instance, the nature photographer Ansel Adams developed methods for selectively overexposing and underexposing the print by waving cardboard cutouts over portions of the print during exposure. While modern image processing offers effective region selection and feathering tools, interactive contrast adjustment has remained a needlessly cumbersome task.

Our aim is to provide a simple, flexible, and precise interactive procedure for specifying the global contrast enhancement of an image. This spatially invariant, global histogram modification operation is performed by a gray level transformation function $T(x)$. At each gray level, its slope determines the change in contrast while its displacement indicates the shift in tone. Simplicity requires that the entire transformation can be easily accomplished by pointing and clicking on the image, so that the user need not be concerned with the shapes of the transformation function and the image histogram. Flexibility requires that contrast can be adjusted simultaneously at more than one point in the tonal range. Precision requires that contrast can be adjusted independently at each chosen point of the tonal range. Our histogram warping method (Figure 1) meets these requirements by using continuously differentiable monotonic splines to express the gray level transformation. 


\section{RELATED WORK}

In image enhancement [1,2] for visual inspection, the role of user interaction in contrast adjustment has received surprisingly little attention. Apart from the numerous automatic algorithms, there are three broad approaches for interactively specifying gray level transformation functions.

The transformation may be defined indirectly through histogram specification [1,3-5]. The user is still faced with the dilemma of selecting the correct histogram for the image. Without taking into account the original histogram, forcing the image to conform to an arbitrary histogram can yield unpredictable results since it is difficult to foresee how much distortion the transformation entails. Moreover, the relationship between the shape of a histogram and the relative contrast of an image may not be readily apparent to an untrained user. A histogram that appears ideal for one image can prove unsuitable for another despite any similarities between the two pictures. Only at most the first three statistical moments of the histogram have been shown to predictably affect contrast [6]. A flat histogram maximizes the entropy of the encoded information while a hyperbolic histogram maximizes the entropy of the perceived brightness [7]. As Gonzalez and Woods [1] observe, "in general, however, there are no rules for specifying histograms".

Alternatively, the gray level transformation may be expressed directly by a mathematical function $y=T(x)$ with its parameters chosen by the user. Since reversing image polarity is not normally desirable, a monotonic increasing transformation $T^{\prime}(x) \geq 0$ is required to preserve the natural order of gray levels. Since abrupt transitions between differing degrees of stretching and compression of the tonal range can cause visible defects, a continuously differentiable $C^{1}$ transformation is required to avoid artificial discontinuities in the new histogram $f\left(T^{-1}(y)\right)\left(T^{\prime}\left(T^{-1}(y)\right)\right)^{-1}$ that results from transforming the original histogram $f(x)$. Our histogram warping technique uses splines designed to meet these two requirements. Previously, contrast enhancement has been performed by linear [1,2,8], quadratic [9], cubic [5,9], sigmoidal [2,10], logarithmic [1], and power law [1,2] functions. Default parameters may be obtained by the optimization of a mathematical criterion [8] or the study of user preference [10]. These simple formulas lack the necessary degrees of freedom to express simultaneous and independent contrast adjustments at different points in the tonal range. Piecewise defined functions can cope with this challenge. Existing implementations fail to meet our requirements, as piecewise exponential [11] and piecewise linear $[1,12]$ histogram transformations are not continuously differentiable while cubic splines [5] can cease to be monotonic in regions of heightened contrast.

Existing image processing packages, such as Adobe Photoshop 7, invite the user to literally draw the gray level transformation curve. As the shape of 

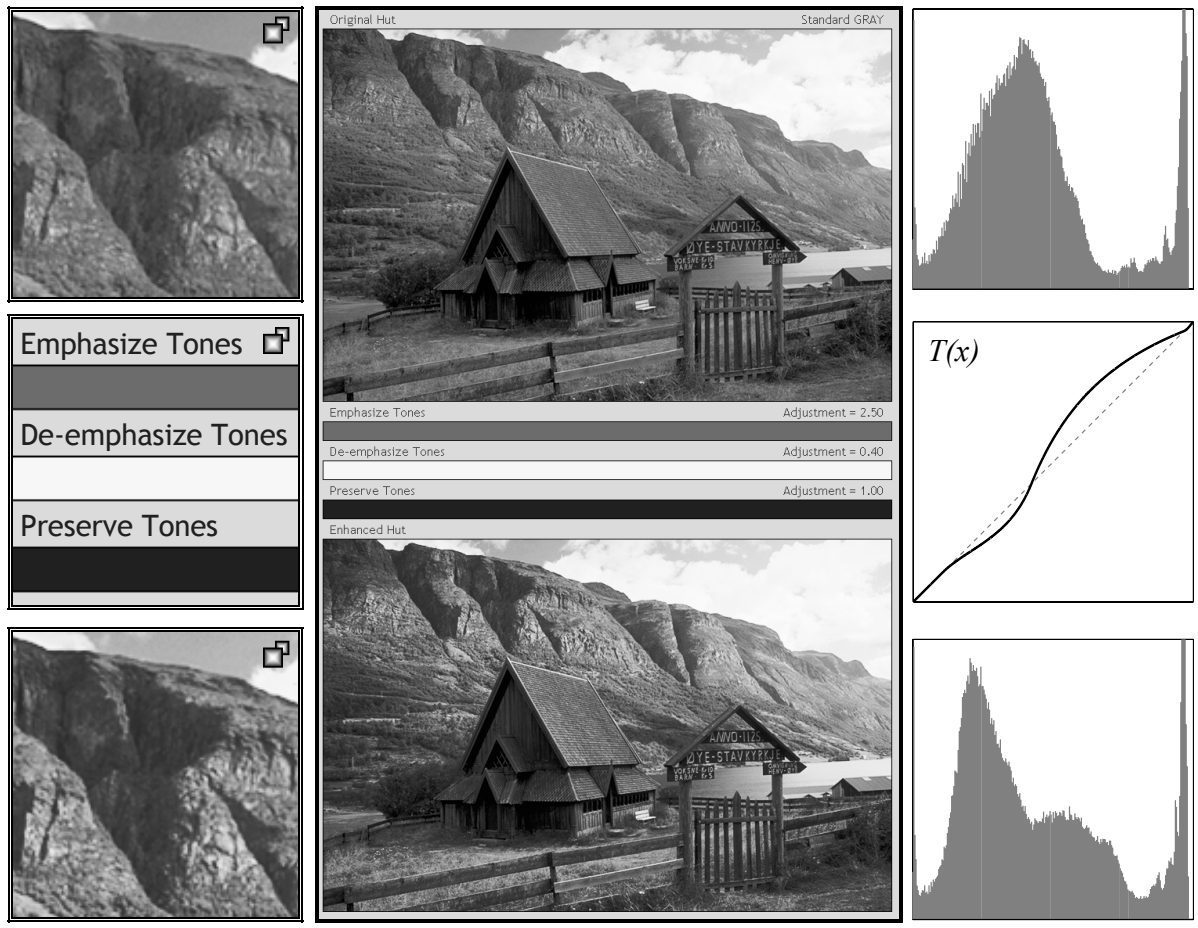

Figure 2. Interactive histogram warping: the original image with its histogram, the contrast adjustments with the gray level transformation, and the resulting image with its histogram.

the curve changes both tone and contrast at the same time, such a user interface demands considerable skill and practice. Instead of focusing on getting the image right, the user must pay attention to getting the curve right. Using design galleries [13] or interactive evolution by aesthetic selection [14] to explore the parameter space [15] of gray level transformations is a plausible alternative, although these approaches to user interaction are usually reserved for applications where direct manipulation does not suffice.

\section{INTERACTION}

A quick way to adjust contrast is to click on the picture. Our user interface (Figure 2, middle) displays the original image alongside the transformed image and a grouped list of contrast adjustments. The contrast adjustment of several key tones can be combined in a single transformation. With the mouse, the user selects the key tones of the original image by clicking on it. Depending on the mouse button, the contrast of a key tone can be raised, lowered, or preserved. The degree of contrast adjustment can be 
set using the arrow keys or the mouse wheel. Acting as anchors, these key tones are preserved by the gray level transformation to ensure that the overall tonal balance of the image is maintained. What changes is the tonal spectrum around these key tones. Where the contrast is raised by increasing $T^{\prime}(x)>1$, the histogram is stretched, and image details of that tone become more prominent. Conversely, where the contrast is lowered by reducing $0 \leq T^{\prime}(x)<1$, the histogram is compressed, and image details become more subdued. Raising the contrast in one region of the tonal range necessitates lowering contrast in another, and vice versa. Where the transformation coincides with the identity mapping $T(x)=x$ and $T^{\prime}(x)=1$, tone and contrast are left unchanged. As more key tones are added, the effect of the contrast adjustments becomes more subtle since the transformation preserves their luminance. Usually a transformation calls for no more than three key tones. A succession of transformations may be applied until the desired effect is achieved. Since the transformations are monotonic and invertible, as long as the gray levels are not quantized, no information is lost. Of course, quantization permits more efficient use of processor and memory resources by discretizing the gray level mapping as a lookup table.

\section{IMPLEMENTATION}

Our histogram warping technique maps gray levels $b_{k}=T\left(a_{k}\right)$ according to their contrast adjustments $d_{k}=T^{\prime}\left(a_{k}\right)$. For monotonicity, we require that the sequence $a_{k}$ is strictly increasing, $b_{k}$ is increasing, and $d_{k}$ is finite and nonnegative. In our application, the key tones $b_{k}=a_{k}$ are preserved and their contrast adjustments $d_{k}$ are determined by the user. The endpoints of the dynamic range may be treated as key tones and, optionally, the contrast of these extreme highlights and shadows may be lowered to raise the contrast of the midtones. Alternatively, the endpoints of the image's tonal range may be remapped to set its white point and its black point. To ensure a continuously differentiable monotonic transformation for any valid choice of inputs, we rely on a piecewise rational quadratic interpolating spline [16,17]:

$$
\begin{gathered}
T(x)=b_{k-1}+\frac{r_{k} t^{2}+d_{k-1}(1-t) t}{r_{k}+\left(d_{k}+d_{k-1}-2 r_{k}\right)(1-t) t}\left(b_{k}-b_{k-1}\right), \\
\text { with } r_{k}=\frac{b_{k}-b_{k-1}}{a_{k}-a_{k-1}} \text { and } t=\frac{x-a_{k-1}}{a_{k}-a_{k-1}} \text { for } x \in\left[a_{k-1}, a_{k}\right] .
\end{gathered}
$$

As future research, it would be interesting to explore more precise control over where the histogram is stretched and compressed by using a piecewise rational cubic interpolating spline with adjustable shape parameters [17]. 


\section{CONCLUSION}

Contrast adjustment is a common user task in digital photography, remote sensing, medical imaging, and scientific visualization. Our technique enables the user to quickly select the key tones of an image and change their contrast without altering their luminance. In future research, we will apply our histogram warping framework to other histogram modification tasks.

\section{REFERENCES}

1. Gonzalez, R. C. and Woods, R. E. 2002. Digital Image Processing, 2 ed. Prentice Hall.

2. Zamperoni, P. 1995. Image Enhancement. Advances in Imaging and Electron Physics, 92, $1-77$.

3. Hummel, R. A. 1975. Histogram Modification Techniques. Computer Graphics \& Image Processing, 4, 3, 209-224.

4. Gonzalez, R. C. and Fittes, B. A. 1977. Gray-Level Transformations for Interactive Image Enhancement. Mechanism \& Machine Theory, 12, 1, 111-122.

5. O'Gorman, L. and Brotman, L. S. 1985. Entropy-Constant Image Enhancement by Histogram Transformation. Proceedings of SPIE, 575, 106-113.

6. Thompson, D. D. and Gonzalez, R. C. 1983. Image Enhancement by Moment Specification. In Proceedings of the 15th Southeastern Symposium on System Theory, 134-137.

7. Frei, W. 1977. Image Enhancement by Histogram Hyperbolization. Computer Graphics \& Image Processing, 6, 3, 286-294.

8. Xu, X. and Miller, E. L. 2002. Entropy Optimized Contrast Stretch to Enhance Remote Sensing Imagery. In Proceedings of 16th International Conference on Pattern Recognition, vol. 3, 915-918.

9. Guo, L. J. 1991. Balance Contrast Enhancement Technique and Its Application in Image Colour Composition. International Journal of Remote Sensing, 12, 10, 2133-2151.

10.Braun, G. J. and Fairchild, M. D. 1999. Image Lightness Rescaling Using Sigmoidal Contrast Enhancement Functions. Journal of Electronic Imaging, 8, 4, 380-393.

11. Raji, A., Thaibaoui, A., Petit, E., et al. 1998. A Gray-Level Transformation-Based Method for Image Enhancement. Pattern Recognition Letters, 19, 13, 1207-1212.

12.Sang-Yeon, K., Dongil, H., Seung-Jong, C., et al. 1999. Image Contrast Enhancement Based on the Piecewise-Linear Approximation of Cdf. IEEE Transactions on Consumer Electronics, 45, 3, 828-834.

13. Marks, J., Andalman, B., Bearsley, P. A., et al. 1997. Design Galleries: A General Approach to Setting Parameters for Computer Graphics and Animation. In Proceedings of SIGGRAPH, 389-400.

14. Sims, K. 1993. Interactive Evolution of Equations for Procedural Models. Visual Computer, 9, 8, 466-476.

15. Taosong, H., Lichan, H., Kaufman, A., et al. 1996. Generation of Transfer Functions with Stochastic Search Techniques. In Proceedings of the 7th IEEE Visualization Conference, 227-234.

16. Gregory, J. A. and Delbourgo, R. 1982. Piecewise Rational Quadratic Interpolation to Monotonic Data. IMA Journal of Numerical Analysis, 2, 123-130.

17. Sarfraz, M., Al-Mulhem, M., and Ashraf, F. 1997. Preserving Monotonic Shape of the Data Using Piecewise Rational Cubic Functions. Computers \& Graphics, 21, 1, 5-14. 